\title{
A Novel Discrete Computational Tool for Microstructure-Sensitive Mechanical Analysis of Composite Materials
}

\author{
Hailong Chen, Yaopengxiao Xu, Yang Jiao, Yongming Liu ${ }^{1}$
}

School for Engineering of Matter, Transport and Energy, Arizona State University, Tempe, AZ, 85287, U.S.A.

\begin{abstract}
In this article, we present a novel pixel-based nonlocal discrete computational tool for the modeling and simulation of mechanical behaviors of composite materials with isotropic phases based on their photographic microstructural information. First, formulation of the proposed model is presented and interface modeling is discussed. Following this, a procedure on quantifying materials effective elastic properties is provided. Details on a spring-based failure criterion for modeling of fracture events are discussed. After that, investigations on effects of material microstructure, interface properties and inclusion characteristics on effective elastic and fracture properties of bi-phase composites reinforced by various inclusions are performed. Based on current study, conclusions are drawn at the end.

Keywords: Image-based Analysis, Lattice Spring Model, Bi-continuous Composites, Fracture, Microstructural Effect
\end{abstract}

\section{Introduction}

With advances of imaging techniques, high-resolution digital micrographs containing quantitative morphological information nowadays are frequently used in many areas, such as mechanics [1], [2], material science [3], [4], optics [5], biology [6] and medicine [7]. Numerous methods exist for generating these digital data: X-ray computed tomography (X-ray CT) [8], scanning electron microscopy (SEM) [9], [10], electron backscatter diffraction (EBSD) [11], magnetic resonance imaging (MRI) [12], to name a few.

* Corresponding author. Tel.: +1 4809656883

E-mail address: Yongming.Liu@asu.edu 
The popularization of digital representation of materials has led to a need for the incorporation of microstructures into numerical materials characterizations.

In last several decades, a great number of approaches have been proposed in the literature to incorporate the microstructural information contained in digital micrographs into numerical models, which can be categorized into two groups: finite element models and discrete element models. In the category of finite element models, such as finite element method [13], [14], [15], [16], extended finite element method [17], [18], finite cell method [19], [20], [21], pixel/voxel-based finite element method [22], [23], [24], [25], [26], the generation of high quality mesh plays significant roles in incorporating the microstructural digital information. In these models, the mesh can be generated either by the pixel/voxel information using externally developed algorithms, such as those described in Ref. [13] - [16], or independent of the pixel/voxel while the phase information is carried by the level set function, as described in Ref. [18]. Given a pixel/voxel based micrograph, a straightforward way that preserves all structural information is to directly taken the pixel/voxel as mesh, as did in Refs. [22] - [26], [19] - [21]. These models inherit all the advantages of finite element method and classical continuum mechanics while efficiently handling the deformation analyses, such as elasticity and plasticity analyses. On the other hand, as these models are still continuum mechanics based, disadvantages of modeling fracture events, such as crack initiation, propagation, branching and coalescence, still persist in models belong to this category. Furthermore, it becomes very challenging to apply these continuum-based models to image-based fracture modeling.

Discrete element models, such as lattice spring models [27], [28], [29], [30], [31], [32], treat pixel/voxel as discrete unit, or particles connected by one dimensional spring element with their corresponding neighbors. From the topological point of view, the lattice spring models share similarity with Pixel/Voxelbased finite element models, since they all directly employ the pixel/voxel into the simulation process 
without any additional mesh generation procedure. For deformation analyses, models in both categories usually can achieve the same accuracy. When applied to study various fracture events, the discrete element models are more beneficial over finite element models. Unlike finite element models, no singularity related issues exist in the discrete models due to their intrinsic nature of discreteness. Another significant advantage of lattice spring models is that fracture processes are natural outcome of the spring breakage. A spring based failure law is sufficient for describing various fracture phenomena [33].

The lattice spring model for mechanical modeling of materials has a long history and can be traced back to the work by Hrennikoff [34]. Nevertheless, it was first applied to image-based mechanical analysis by Day and coworkers in early 1990's [27], [28]. As has been pointed out later by Day [23], the lattice spring model proposed in [27] and [28] only applicable to certain materials: (1) the materials Poisson's ratios were required to be no less than $1 / 3$; and (2) material geometry was digitally represented using hexagonal pixels on triangular lattice. Later, Pazdniakou et al. [29] applied the 3D cubic lattice spring model to study the dynamic behavior of reconstructed porous media. The limitation on materials' Poisson's ratio also exists in [29].

The objective of current work is to overcome the limitation on Poisson's ratio and extend the application of discrete element based lattice spring models to microstructure sensitive fracture behavior analysis based on material micrographs. A nonlocal discrete lattice spring model developed in [33] is applied to image-based materials characterization. The nonlocality of the interaction between pixels lies in the fact that it not only depends on the connecting spring but also has contribution from all its neighboring springs. The content of this paper is organized as follow. First, a detailed theoretical formulation of the nonlocal lattice spring model is given. The model parameters are derived based on energy equivalency and theory of hyper-elasticity. Following this, interfaces between different material phases are modeled 
and homogenization procedures are discussed. A spring-based critical elongation failure criterion is proposed for fracture modeling. Next, numerical results for homogenization analysis of elastic properties and biaxial fracture simulation are presented. Finally, conclusions and future work are drawn based on this study.

\section{Theory and formulation}

In 2D image-based analysis, the physical microstructure is represented as an array of pixels. For multiphase microstructure, the phase information is usually stored as assigned value associated with each pixel. Different values are used to indicate different phases such that each phase is distinctive from other phases. The proposed computational tool is completely based on this photographic information.

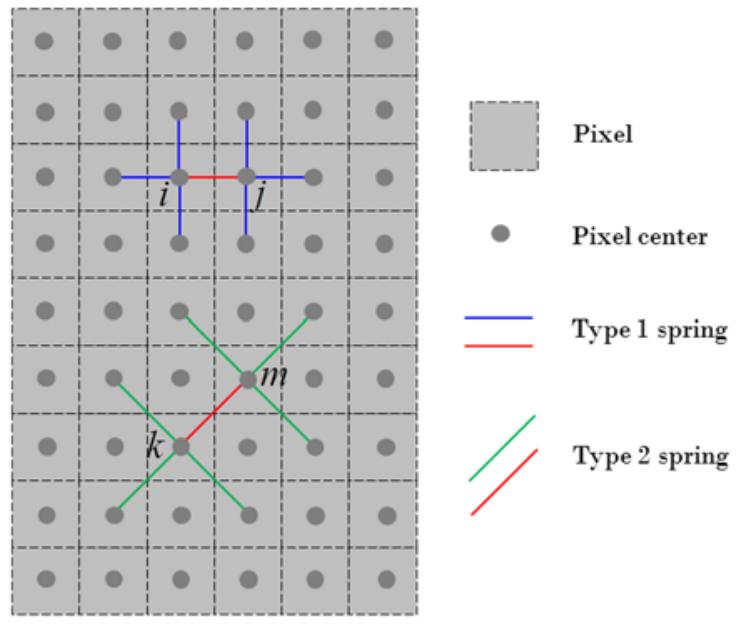

Figure 1. The nonlocal interaction between a typical pixel pair

Before modeling responses of microstructure under external mechanical loadings, interactions between pixels via axial springs need to be determined. In the propose model, the interaction between a typical pixel pair not only dependents on the pixel pair itself, but also has contribution from pixels surrounding them, see Fig. 1. For pixel $i$, its interaction with the first nearest neighboring pixel $j$ is determined by the connecting type 1 spring (red type 1 spring) and all surrounding springs connecting with the first nearest neighboring pixels of pixel $i$ and $j$ (blue type 1 spring). The same applies to the interaction with the 
second and up to the $n$th nearest neighboring pixels. In this study, only the first and second neighboring pixels are considered as active neighbors of the center reference pixel, i.e., interaction only exist between the center reference pixel and its first and second neighboring pixels.

In order to account for the interaction between each pixel pair, the concept of unit cell is used for this purpose. A unit cell is defined as the repeating unit identified based the pixel and its neighbors of the same type in the physical domain. For this two-neighbor case, the identified unit cells for different type of neighbors are shown in Fig. 2. The corresponding unit normal vectors for different unit cells are given in Table 1.

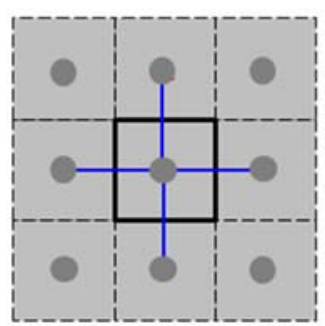

unit cell 1

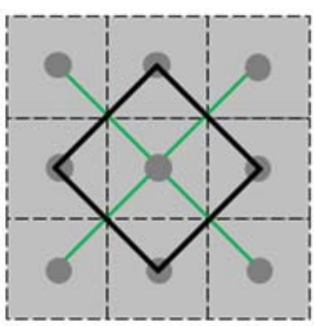

unit cell 2

Figure 2. The unit cell for each type of neighboring pixels

Table 1. List of normal vectors for two unit cells

\begin{tabular}{cccc}
\hline \multicolumn{5}{c}{ neighbor 1 $\left(N_{1}=4\right)$} \\
\hline$n_{1}$ & $(1,0,0)$ & $n_{3}$ & $(0,1,0)$ \\
$n_{2}$ & $(-1,0,0)$ & $n_{4}$ & $(0,-1,0)$ \\
\hline$n_{5}$ & $(\sqrt{2} / 2, \sqrt{2} / 2,0)$ & $n_{7}$ & $(\sqrt{2} / 2,-\sqrt{2} / 2,0)$ \\
$n_{6}$ & $(-\sqrt{2} / 2,-\sqrt{2} / 2,0)$ & $n_{8}$ & $(-\sqrt{2} / 2, \sqrt{2} / 2,0)$ \\
\hline
\end{tabular}

The derivation of spring parameters follows the same procedure as in [35]. But different from the classical lattice spring model [36], [37], the proposed model introduces an additional nonlocal potential in terms of axial springs [38]. 
The potential of a typical pixel is the sum of potentials of two unit cells,

$$
U_{\text {pixel_ } i}=\sum_{k=1}^{2} U_{\text {cell_k }}^{i}
$$

and for each unit cell it has two parts as

$$
U_{\text {cell_k }}^{i}=U_{\text {local_k }}^{i}+U_{\text {nonlocal_k }}^{i}
$$

with

$$
\begin{aligned}
& U_{\text {local_k }}^{i}=\frac{1}{2}\left(k_{k}^{i} \sum_{j=1}^{N_{k}^{i}}\left(\delta I_{k j}^{i}\left\lceil\delta I_{k j}^{i}\right)\right)\right. \\
& U_{\text {nonlocal_k }}^{i}=\frac{1}{2} t_{k}^{i}\left(\sum_{j=1}^{N_{k}^{i}} \delta I_{k j}^{i}\right)\left(\sum_{j=1}^{N_{k}^{i}} \delta I_{k j}^{i}\right)
\end{aligned}
$$

where $k_{k}^{i}$ and $t_{k}^{i}$ are the local and nonlocal parameters for the axial springs of unit cell $k$ of pixel $i, \delta l_{k j}^{i}$ is the half length of the elongation of spring $j$ for unit cell $k$ of pixel $i, N_{k}^{i}$ is the total number of active neighboring pixels for unit cell $k$ of pixel $i$.

Mapping directional spring elongations to the strain tensor using normal vectors in Table 1 , the potential of a unit cell can be rewritten as

$$
U_{\text {cell_k }}^{i}=\frac{1}{8}\left(I_{k}\right)^{2}\left(k_{k}^{i} \sum_{b=1}^{N_{k}^{i}} n_{l}^{b} \varepsilon_{I J} n_{J}^{b} n_{k}^{b} \varepsilon_{K L} n_{L}^{b}+t_{k}^{i}\left(\sum_{b=1}^{N_{k}^{i}} n_{l}^{b} \varepsilon_{I J} n_{J}^{b}\right)\left(\sum_{b=1}^{N_{k}^{i}} n_{k}^{b} \varepsilon_{K L} n_{L}^{b}\right)\right)
$$

where $I_{k}$ is the distance between the reference pixel $i$ with its $k$ th nearest neighboring pixel, i.e., the original spring length. $I_{1}$ equals to a pixel length, and $I_{2}$ is $\sqrt{2}$ times of a pixel length.

For each pixel, the total potential is conservative for elastic materials. Thus, equating the potential to a continuum counterpart, the material stiffness tensor can be obtained according to Theory of Hyperelasticity as 


$$
\begin{aligned}
C_{I J K L}^{i} & =\frac{1}{V_{1}} \frac{\partial^{2}\left(U_{c e l l}^{i} 1\right)}{\partial \varepsilon_{I J} \partial \varepsilon_{K L}}+\frac{V_{2}}{V_{1}}\left(\frac{1}{V_{2}} \frac{\partial^{2}\left(U_{c e l I_{-2}}^{i}\right)}{\partial \varepsilon_{I J} \partial \varepsilon_{K L}}\right) \\
& =\frac{1}{8} k_{1}^{i} \sum_{b=1}^{4} n_{l}^{b} n_{J}^{b} n_{K}^{b} n_{L}^{b}+\frac{1}{8} t_{1}^{i}\left(\sum_{b=1}^{4} n_{l}^{b} n_{J}^{b}\right)\left(\sum_{b=1}^{4} n_{K}^{b} n_{L}^{b}\right)+\frac{1}{4} k_{2}^{i} \sum_{b=5}^{8} n_{l}^{b} n_{J}^{b} n_{K}^{b} n_{L}^{b}+\frac{1}{4} t_{2}^{i}\left(\sum_{b=5}^{8} n_{l}^{b} n_{J}^{b}\right)\left(\sum_{b=5}^{8} n_{K}^{b} n_{L}^{b}\right)
\end{aligned}
$$

where $V_{1}=h\left(I_{1}\right)^{2}$ is the volume of unit cell 1 , and $V_{2}=h\left(I_{2}\right)^{2}$ is the volume of unit cell 2 . For consistency, the unit thickness $h$ is used. In following content, the unit thickness $h$ is omitted unless confusion arises.

Plugging in unit normal vectors given in Table 1, the elastic stiffness matrix can be obtained after simplification as

$$
\left[C^{i}\right]=\left[\begin{array}{ccc}
k_{1}^{i} / 2+t_{1}^{i}+k_{2}^{i} / 2+2 t_{2}^{i} & t_{1}^{i}+k_{2}^{i} / 2+2 t_{2}^{i} & 0 \\
t_{1}^{i}+k_{2}^{i} / 2+2 t_{2}^{i} & k_{1}^{i} / 2+t_{1}^{i}+k_{2}^{i} / 2+2 t_{2}^{i} & 0 \\
0 & 0 & k_{2}^{i} / 2
\end{array}\right]
$$

For isotropic materials, assuming nonlocal parameters for two unit cells are identical, model parameters in terms of materials Young's modulus and Poisson's ratio can be uniquely solved as for plane strain case:

$$
\begin{aligned}
& k_{1}^{i}=\frac{2 E^{i}}{1+v^{i}}, \quad k_{2}^{i}=\frac{E^{i}}{1+v^{i}} \\
& t^{i}=\frac{E^{i}\left(4 v^{i}-1\right)}{6\left(1+v^{i}\right)\left(1-2 v^{i}\right)}
\end{aligned}
$$

for plane stress case:

$$
\begin{aligned}
& k_{2}^{i}=\frac{2 E^{i}}{1+v^{i}}, \quad k_{2}^{i}=\frac{E^{i}}{1+v^{i}} \\
& t^{i}=\frac{E^{i}\left(3 v^{i}-1\right)}{6\left(1+v^{i}\right)\left(1-v^{i}\right)}
\end{aligned}
$$


Given the potential in Eqs. (1) - (4), the interaction between each pixel pair can be obtained by differentiating the potential with respect to the corresponding spring elongation as

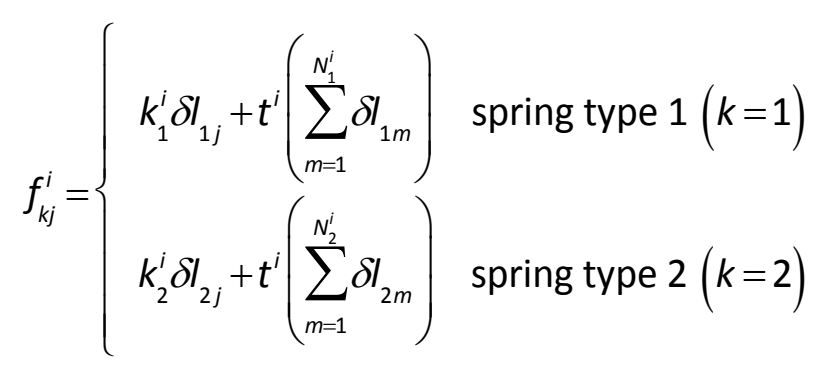

Rewrite above Eq. (10), the effective spring stiffness parameter can be formulated as

$$
k_{k j}^{i}=\left\{\begin{array}{l}
k_{1}^{i}+t^{i}\left(\sum_{m=1}^{N_{1}^{i}}\left(\frac{\delta l_{1 m}}{\delta l_{1 j}}\right)\right) \text { spring type 1 } \\
k_{2}^{i}+t^{i}\left(\sum_{m=1}^{N_{2}^{i}}\left(\frac{\delta l_{2 m}}{\delta l_{2 j}}\right)\right) \quad \text { spring type 2 }
\end{array}\right.
$$

The effective spring stiffness is nonlocal. It could be different for springs connecting different pixels.

The stress tensors at the pixel center can be calculated as

$$
\begin{aligned}
& \sigma^{i}=\frac{1}{V_{1}} \frac{\partial U_{c e l l_{-} 1}^{i}}{\partial \varepsilon^{i}}+\frac{V_{2}}{V_{1}}\left(\frac{1}{V_{2}} \frac{\partial U_{c e l I_{2} 2}^{i}}{\partial \varepsilon^{i}}\right)=\frac{1}{V_{1}} \frac{\partial\left(U_{\text {cell_1 }}^{i}+U_{c e l I_{-} 2}^{i}\right)}{\partial \varepsilon^{i}} \\
& =\frac{1}{V_{1}} \sum_{k=1}^{2} \sum_{j=1}^{N_{k}^{i}} \frac{\partial U_{c e l l \_k}^{i}}{\partial \delta I_{k j}} \frac{\partial \delta I_{k j}}{\partial \varepsilon^{i}}=\frac{1}{V_{1}} \sum_{k=1}^{2} I_{k} \sum_{j=1}^{N_{k}^{i}} \frac{\partial U_{c e l I_{-} k}^{i}}{\partial \delta I_{k j}} \frac{\partial e_{k j}}{\partial \varepsilon^{i}} \\
& =\frac{1}{V_{1}} \sum_{k=1}^{2} I_{k} \sum_{j=1}^{N_{k}^{i}} \frac{\partial U_{c e l l}^{i}{ }_{1} k}{\partial \delta I_{k j}} \mathbf{n}_{k j}\left(\mathbf{n}_{k j}\right)^{T}=\frac{1}{V_{1}} \sum_{k=1}^{2} I_{k} \sum_{j=1}^{N_{k}^{i}}\left(k_{k}^{i} \delta I_{k j}+t^{i}\left(\sum_{m=1}^{N_{k}^{i}} \delta I_{k m}\right)\right) \mathbf{n}_{k j}\left(\mathbf{n}_{k j}\right)^{T}
\end{aligned}
$$

where $e_{k j}$ is the directional strain, i.e. $e_{k j}=\frac{\delta I_{k j}}{I_{k}}$.

The strain tensor then can be obtained using

$$
\boldsymbol{\varepsilon}^{i}=\mathbf{S}^{i} \cdot \boldsymbol{\sigma}^{i}
$$

where $\mathbf{S}^{i}$ is the compliance matrix. 


\section{The interface}

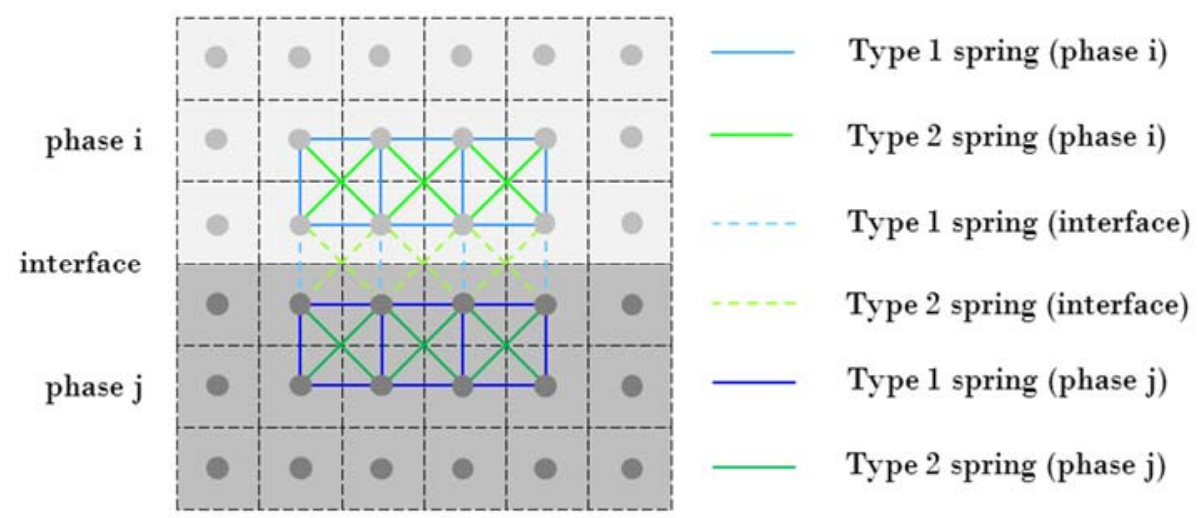

Figure 3. Representation of the interface in lattice spring model

Interface intrinsically exists in lattice spring models, represented by springs straddling two different phases, as shown in Fig. 3. Thus, by assigning different properties to these springs, the role of interfaces can be effective considered. The properties of these interface springs can be determined through experiments [39], Molecular Dynamics simulation [40], [41], and some averaging algorithms [42].

\section{Estimation of effective elastic constants}

Procedure on estimating effective elastic constants in the proposed model is developed based on the system strain energy. Similar procedures can be found in [43] and [44]. A two-step estimation scheme is outlined as follows:

(i) Subjecting the microstructure to a uniform bi-axial extension. The corresponding strain state is $\varepsilon_{x x}=\varepsilon_{y y}=\varepsilon_{0}, \varepsilon_{x y}=0$. The total energy $U_{(1)}$ is the sum of energies of all pixels;

(ii) Subjecting the microstructure to uniform uniaxial extension along one direction and to uniform uniaxial compression along the perpendicular direction. The corresponding strain state is $-\varepsilon_{11}=\varepsilon_{22}=\varepsilon_{0}, \varepsilon_{x y}=0$. The total energy of the system is $U_{(2)}$. 
In terms of bulk modulus $K$ and shear modulus $G$, the energy of a two-dimensional linear elastic isotropic continuum of area $A$ is

$$
U=A\left(\frac{K}{2} \varepsilon_{i i} \varepsilon_{j j}+G\left(\varepsilon_{i j} \varepsilon_{i j}-\frac{1}{2} \varepsilon_{i i} \varepsilon_{j j}\right)\right)
$$

Thus, the two-dimensional bulk and shear moduli can be calculated through these steps as

$$
\begin{aligned}
& K=\frac{1}{2} \frac{U_{(1)}}{A \varepsilon_{0}^{2}} \\
& G=\frac{1}{2} \frac{U_{(2)}}{A \varepsilon_{0}^{2}}
\end{aligned}
$$

For two-dimensional linear elastic isotropic materials, bulk and shear moduli can be expressed in terms of Young's modulus and Poisson's ratio as [45]

$$
G=\frac{E}{2(1+v)}
$$

for plane stress case, and

$$
K=\frac{E}{2(1-v)}
$$

for plane strain case,

$$
K=\frac{E}{2(1+v)(1-2 v)}
$$

\section{Spring-based failure criterion}

Other than stress or energy based failure criteria in continuum fracture mechanics, a spring-based critical elongation failure criterion is utilized to simulation fracturing problems in the proposed model. Some other bond-based failure criteria are available in the literature, such as critical force [38]. The advantage of spring-based failure criteria is that only localized spring breakage condition is required for 
fracture simulation. No need of rules for crack propagation, branching and coalescence. And these events are the naturally outcome of spring breakage.

The critical elongation criterion in the proposed model has the following form:

$$
\delta l_{i}^{\text {critical }}=\alpha_{i} \cdot \delta l_{i}^{0}
$$

with $i=1,2$ for spring connecting with the first and second neighbors, $\delta l_{i}^{0}$ is the original spring length, and $\alpha_{i}$ is the critical spring strain, which is usually calibrated from experimental data.

By vector projection, the critical spring strains for first neighbors and second neighbors of a pixel should have following relationship for isotropic materials

$$
\alpha_{1}=\frac{\alpha_{2}}{\sqrt{2}}
$$

Using the above derived relationship between the critical spring strains for these two types of springs, there is only one parameter needs to be calibrated from experimental data.

The simulation of cracking events in the proposed model is carried out as follows: at each simulation time step, the elongation of springs are calculated and compared with corresponding critical values. If calculated elongation is larger than critical value, then the spring is considered as broken and it cannot sustain any tensile force in following simulation steps. This process continues until simulation ends.

\section{Numerical results}

In this section, several numerical results obtained using the proposed pixel-based lattice spring model are presented, including the effective elastic constants of a bi-continuous composite and the fracture characteristics of composite materials reinforced with circular and square inclusions. 


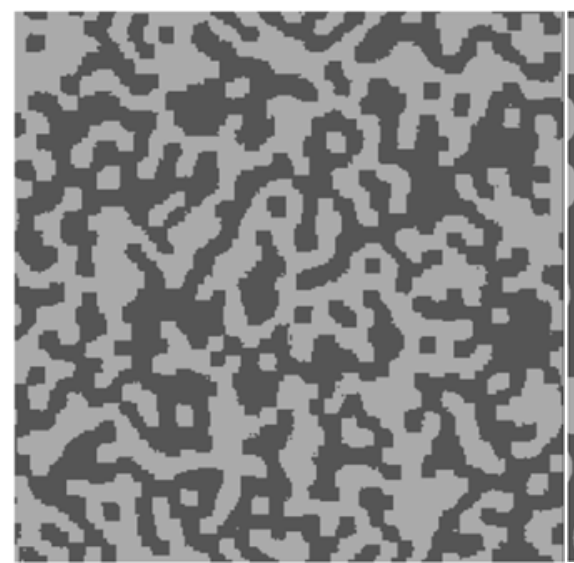

(a). Bi-continuous case

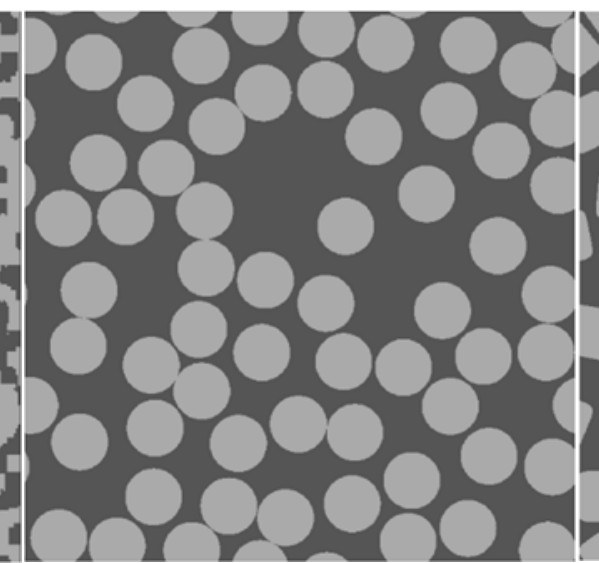

(b). Circular case

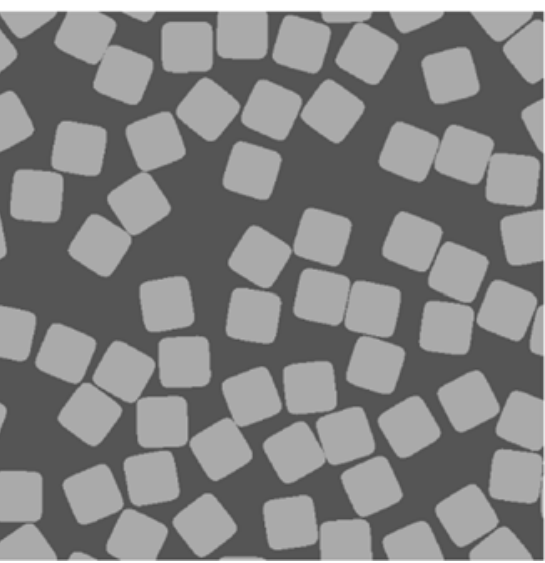

(c). Square case

Figure 4. Microstructures of various bi-phase composites with $50 \%$ volume fraction

Rather than real digital data, the microstructural systems used in this study are generated using the hard-particle Monte Carlo (MC) simulations [46]. Specifically, certain numbers of small non-overlapping inclusions, i.e., particulates in this study, are initially placed in a simulation box with random positions and orientations. At each MC stage, the inclusions are given random translations and rotations, subject to the non-overlapping constraints. At the end of the $M C$ stage, attempts are made to shrink the simulation box without causing overlap between any pairs of inclusions. This process is repeated until the desired volume fraction is achieved. A typical system generated using the hard-particle Monte Carlo simulation is shown in Fig. 4, with different reinforcements. Simulation in this section will base on these microstructures.

\subsection{Effective elastic constants}

The estimation of effective elastic properties of random, multiphase materials is an old, challenging and important problem with applications in almost every field of materials technology. Extensive works, both analytical and numerical, have been done in this field, such as [47], [48], [49]. In this subsection, the proposed pixel-based lattice spring model is applied to estimate the effective elastic properties of a bi-continuous composite, as shown in Fig. 4(a). The dimension is assumed to be $0.01 \mathrm{~m}$. For simplicity, the elastic properties of the two constituent materials are assumed to be: $E_{m}=110 \mathrm{GPa}, v_{m}=0.34$ and 
$E_{i}=450 \mathrm{GPa}, v_{i}=0.17$, where $m$ indicates the matrix and $i$ for the inclusion. Two types of interface properties are used, i.e., interface 1 assumes the interface has the same properties as the inclusion while interface 2 assigns matrix properties to the interface. As has been mentioned in Section 3, some other values can be assigned to the interface.

The procedure presented in Section 4 has been carried out to estimate the effective values of elastic constants. The obtained results are shown in Fig. 5, compared with Hashin-Shtrikman (H-S) bound [48]. As can be seen, for interface 1, i.e., the interface has the same properties as the matrix, the predicted effective constants, bulk modulus and shear modulus, fall within the $\mathrm{H}-\mathrm{S}$ bounds for all inclusion volume fraction from 0.0 to 1.0 . While for the interface 2 , i.e., the interface properties are the same as the inclusion, the predicted effective constants are less than the H-S lower bounds for inclusion volume fraction 0.0 to 0.5 , and becomes within the $\mathrm{H}-\mathrm{S}$ bounds for volume fraction 0.5 to 1.0 . This is due to the fact that the interface becomes softer in this case. The calculated strain and stress components using the Eqs (12) and (13) for the case of 50\% volume fraction are shown in Fig. 6.
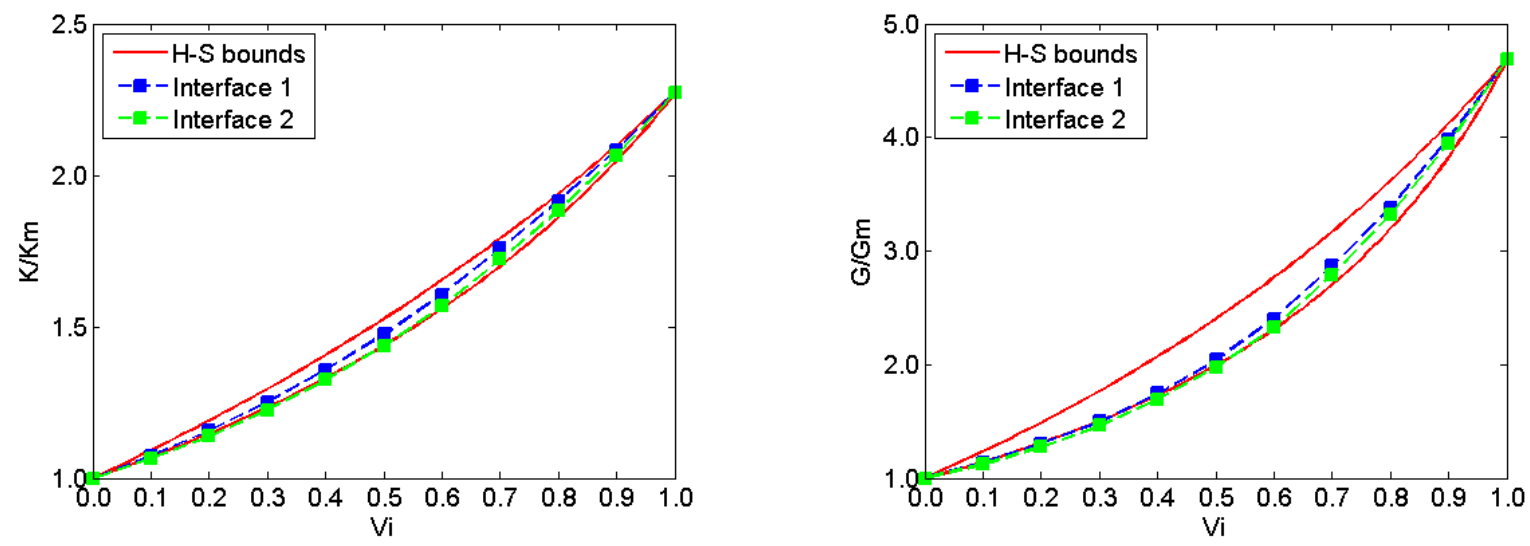

Figure 5. The predicted elastic constants for bi-continuous microstructure 

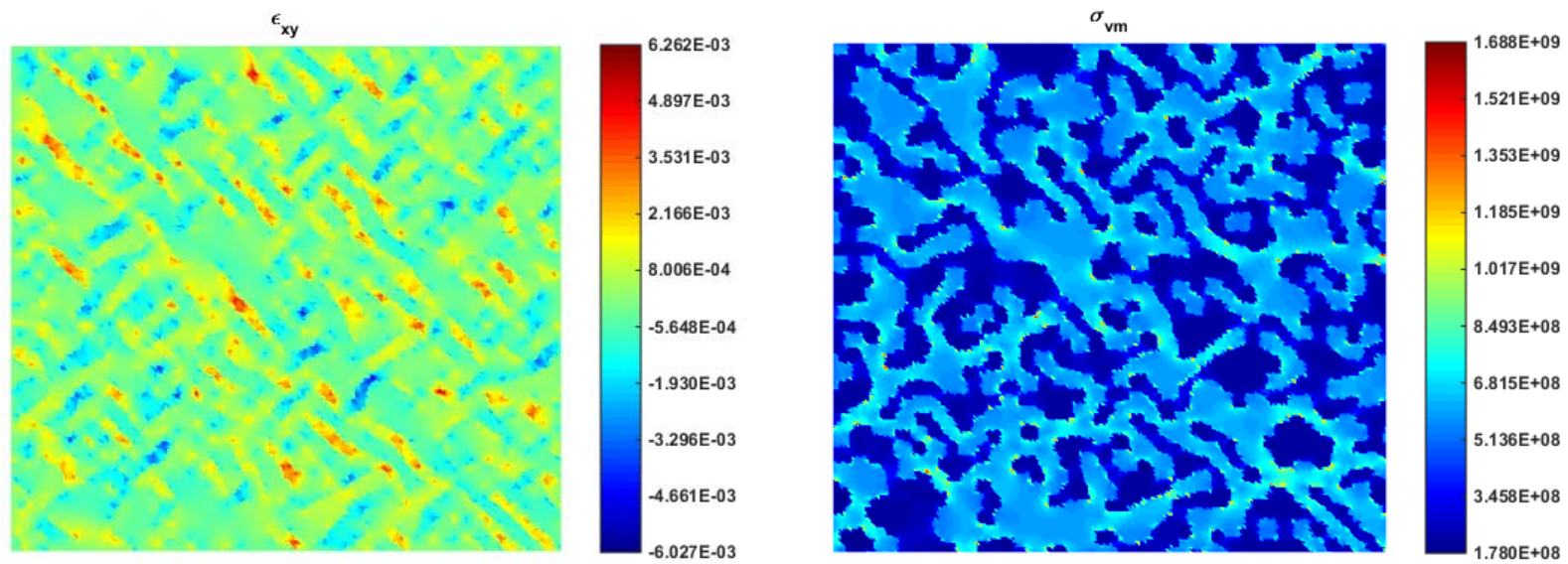

Figure 6. The strain $\varepsilon_{x y}$ and von Mises stress $\sigma_{v m}$ distributions within the composites $(v o l \%=50)$

Over all, predicted results are consistent with H-S analytical predictions. One advantage of the proposed model or any other numerical model over analytical predictions is that besides the effective elastic properties, they can lead to detailed information of the associated stress and strain field within the materials with arbitrary microstructures under random loadings. Such information cannot be obtained from theoretical analysis.

\subsection{Crack initiation and propagation}

As has been mentioned in the Introduction and Section 5, lattice spring models are advantageous over continuum models for fracture modeling. And only a spring-based failure criterion is required for different fracture events. In this subsection, the nonlocal lattice spring model is applied to study the crack initiation and propagation behaviors of bi-phase composites reinforced with $50 \%$ circular and rectangular inclusions. The microstructures have the same dimension and properties as in example 6.1, except that the Young's modulus is $E_{i}=45 \mathrm{GPa}$ for the case of soft inclusion. A bi-axial loading scenario is simulated by applying displacement boundary conditions on all four edges pulling outward. For simplicity, the critical spring strain was assumed to be $\alpha_{1}=0.01, \alpha_{2}=\frac{0.01}{\sqrt{2}}$. The spring is considered to be broken and cannot sustain any tensile force as long as the spring elongation is detected to be 
larger than the specified critical value. Interfaces in all simulation are assumed to have the matrix properties.

The simulation results for the circular inclusion are shown in Fig. 7, with (a) and (b) for hard inclusion and (c) and (d) for soft inclusion. The white lines indicate the crack path. The initiation positions are highlighted. As can be seen, for fixed boundary condition but different inclusion property, the stress distribution within the material is different, thus the crack initiation location is different, see Fig. $7(a)$ and Fig. 7(c). The corresponding material strengths are $1169 \mathrm{MPa}$ and $279 \mathrm{MPa}$ for hard and soft inclusions, respectively. For hard inclusion, the crack path avoids the inclusion and propagates along the interface and through the weak material. For soft inclusion, since interfaces were assumed to have the same properties as the matrix, the crack tends to grow through the soft inclusions, but avoid interface and matrix.

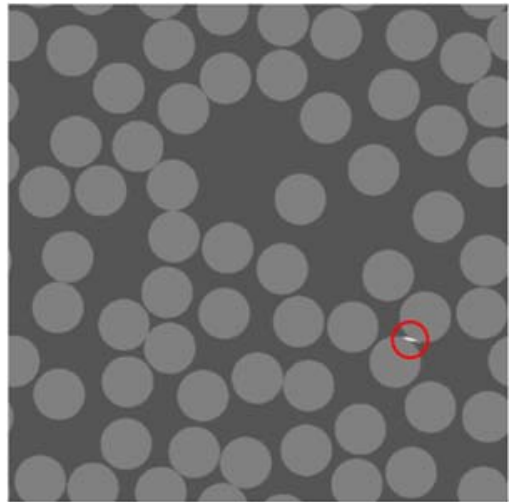

(a) crack initiation for hard inclusion

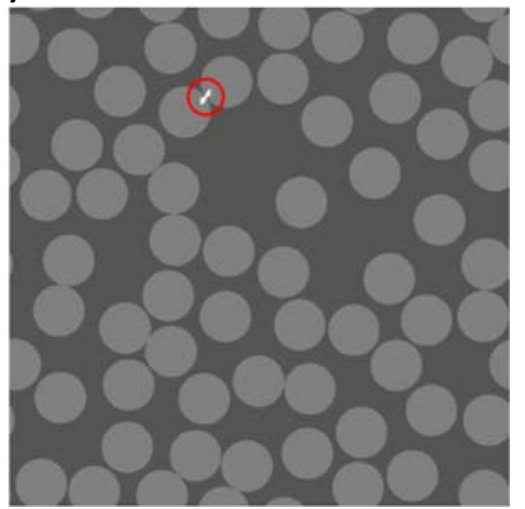

(c) crack initiation for soft inclusion

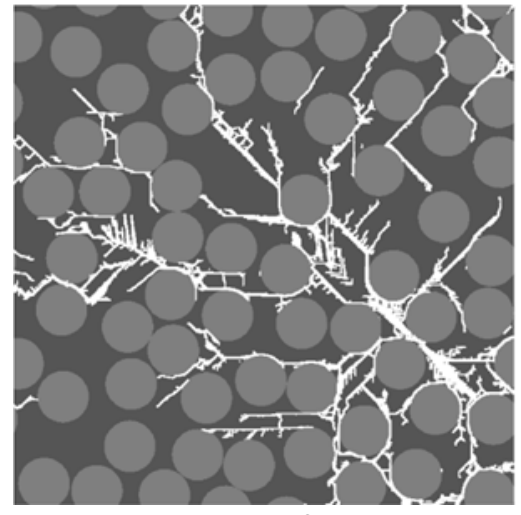

(b) crack propagation for hard inclusion

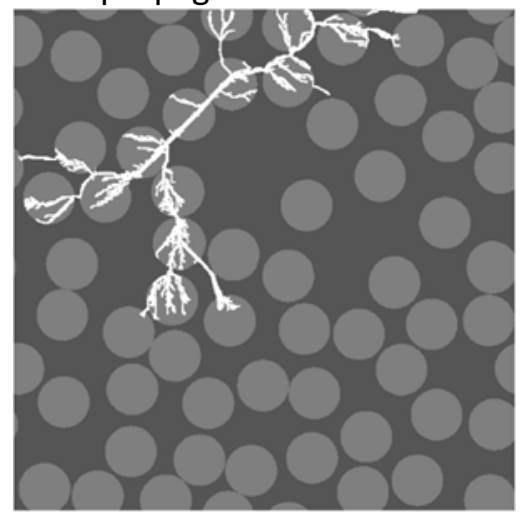

(d) crack propagation for soft inclusion

Figure 7. The predicted crack initiation and propagation for circular inclusion 
The simulation results for the square inclusion are shown in Fig. 8, with (a) and (b) for hard inclusion and (c) and (d) for soft inclusion. Similar to the circular inclusion, the crack initiation location and propagation path both are different for different inclusion properties. The corresponding material strengths are $1029 \mathrm{MPa}$ and $288 \mathrm{MPa}$ for hard and soft inclusions, respectively.

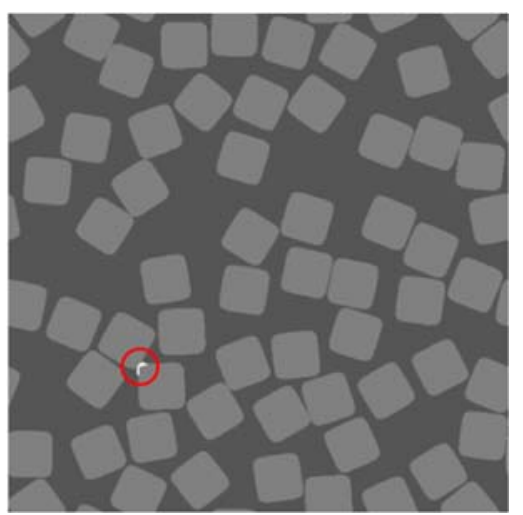

(a) crack initiation for hard inclusion

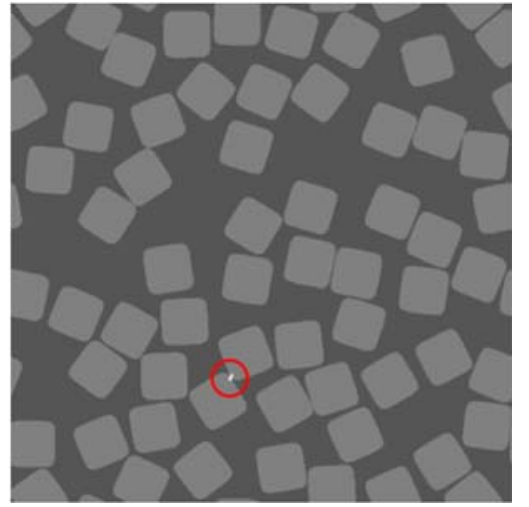

(c) crack initiation for soft inclusion

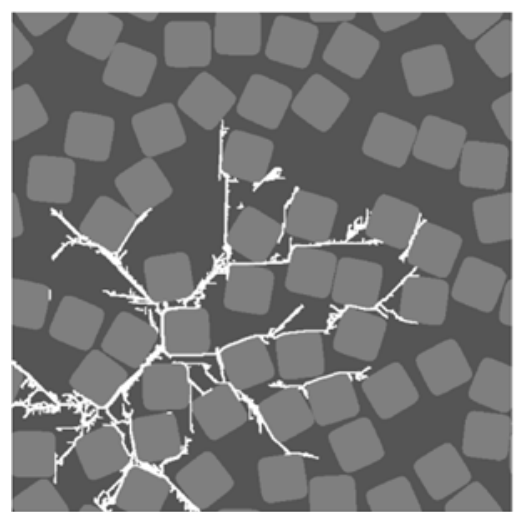

(b) crack propagation for hard inclusion

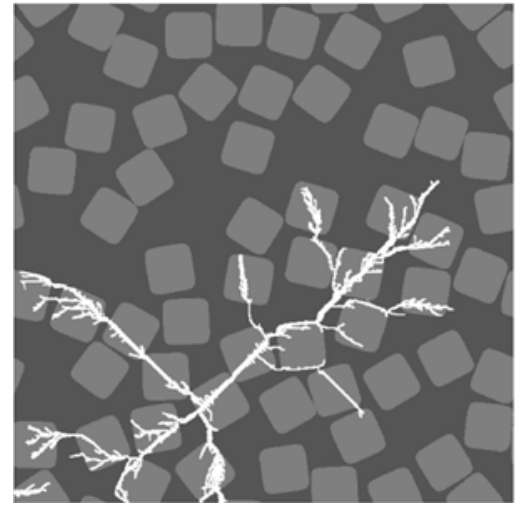

(d) crack propagation for soft inclusion

Figure 8. The predicted crack initiation and propagation for square inclusion

With the same volume fraction and inclusion number, the inclusion shape, i.e., circular or square, doesn't show significant influence on the material strength. Further study is required to investigate the inclusion distribution effect.

The load-displacement curves for various inclusion shapes and properties are shown in Fig. 9. Due to the assigned brittle behavior of each connecting spring, the correlation between the microscopic events and the macroscopic response is not obvious from curves shown in Fig. 9. 


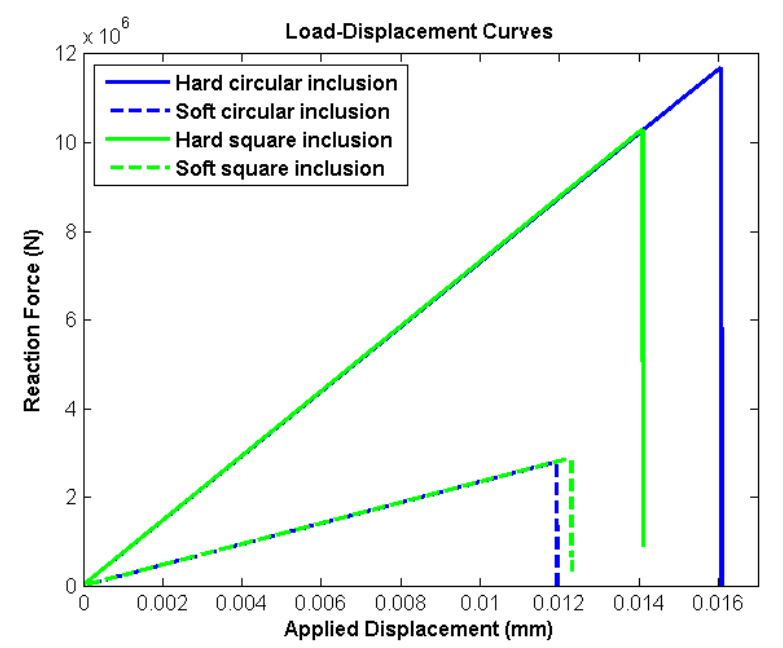

Figure 9. The load-displacement curves for various inclusion cases

\section{Conclusion and future work}

A novel nonlocal discrete computational tool has been developed for pixel-based materials modeling and simulation. In the proposed framework, pixels were directly incorporated into the modeling and simulation process, which interact with them up to the second neighbors. A nonlocal inter-pixel potential was proposed to account interactions between neighboring pixels, whose parameters are determined by energy equivalency with its continuum counterpart and the theory of hyper-elasticity. The resulted pixel interaction is nonlocal in the sense that it not only depends on deformation of the connecting spring, but also has contribution from all neighboring springs. Interface was modeled by assigning different model parameters to springs straddling two different phases. Energy based numerical homogenization procedure was outlined and a spring-based critical elongation failure criterion was discussed. Digital microstructure data that generated using the hard-particle Monte Carlo simulation were used in the modeling and simulation. For homogenization of a bi-continuous composite, the predicted effective elastic constants with different interface were consistent with Hashin-Shtrikman bounds. For fracture modeling of bi-phase composites reinforced with circular and square particles, the crack initiations and propagations can be captured using the spring-based failure criterion. Detailed model validation with experimental measurements needs further study. 
The advantage of the proposed image-based computational tool for fracture modeling over other continuum mechanics based models is very obvious. Both tensile failure and shear failure can be simulated using the spring based critical elongation criterion. Given a spring failure elongation, the simulated tensile failure strength is different from the shear strength. The validity of a single spring failure criterion for two independent failure behaviors, i.e. tensile failure and shear failure, needs further study. Future work also includes systematic study of various fracture events using the proposed tool in image-based analysis. Extension of current framework to 3D voxel-based materials modeling and simulation needs additional study.

\section{Acknowledgement:}

This work is partially supported by DARPA under grant No. N66001-14-1-4036. 


\section{References}

1. Kujime, T., et al., Three-dimensional image-based modeling of lotus-type porous carbon steel and simulation of its mechanical behavior by finite element method. Materials Science and Engineering: A, 2007. 460-461: p. 220-226.

2. Thomas, J., M. Groeber, and S. Ghosh, Image-based crystal plasticity FE framework for microstructure dependent properties of Ti-6Al-4V alloys. Materials Science and Engineering: $A$, 2012. 553: p. 164-175.

3. Jiao, Y., E. Padilla, and N. Chawla, Modeling and predicting microstructure evolution in lead/tin alloy via correlation functions and stochastic material reconstruction. Acta Materialia, 2013. 61(9): p. 3370-3377.

4. Li, H., N. Chawla, and Y. Jiao, Reconstruction of heterogeneous materials via stochastic optimization of limited-angle X-ray tomographic projections. Scripta Materialia, 2014. 86: p. 4851.

5. $\quad$ Débarre, D., et al., Image-based adaptive optics for two-photon microscopy. Optics letters, 2009. 34(16): p. 2495-2497.

6. Phooi Yee, L. and S. Ozawa. An image-based analysis for classifying multimodal brain images in the Image-guided Medical Diagnosis Model. in Engineering in Medicine and Biology Society, 2004. IEMBS '04. 26th Annual International Conference of the IEEE. 2004.

7. Koch, R.G., et al., A Custom Image-Based Analysis Tool for Quantifying Elastin and Collagen Micro-Architecture in the Wall of the Human Aorta from Multi-Photon Microscopy. Journal of biomechanics, 2014. 47(5): p. 935-943.

8. Stiénon, A., et al., A new methodology based on X-ray micro-tomography to estimate stress concentrations around inclusions in high strength steels. Materials Science and Engineering: A, 2009. 513-514: p. 376-383.

9. Klein, T., E. Buhr, and C. Georg Frase, Chapter 6 - TSEM: A Review of Scanning Electron Microscopy in Transmission Mode and Its Applications, in Advances in Imaging and Electron Physics, W.H. Peter, Editor. 2012, Elsevier. p. 297-356.

10. Cantrell, J.H. and M. Qian, Scanning electron acoustic microscopy of SiC particles in metal matrix composites. Materials Science and Engineering: A, 1989. 122(1): p. 47-52.

11. Schwartz, A.J., et al., Electron Backscatter Diffraction in Materials Science. 2nd ed. 2009, New York: Springer.

12. Kosy, J., V. Mandalia, and R. Anaspure, Characterization of the anatomy of the anterolateral ligament of the knee using magnetic resonance imaging. Skeletal Radiology, 2015. 44(11): p. 1647-1653.

13. Zohdi, T.I., P. Wriggers, and C. Huet, A method of substructuring large-scale computational micromechanical problems. Computer Methods in Applied Mechanics and Engineering, 2001. 190(43-44): p. 5639-5656.

14. Langer, S.A., E.R. Fuller, Jr., and W.C. Carter, OOF: an image-based finite-element analysis of material microstructures. Computing in Science \& Engineering, 2001. 3(3): p. 15-23.

15. Mishnaevsky Jr, L., U. Weber, and S. Schmauder, Numerical analysis of the effect of microstructures of particle-reinforced metallic materials on the crack growth and fracture resistance. International Journal of Fracture, 2004. 125(1): p. 33-50.

16. Reid, A.C.E., et al., Image-based finite element mesh construction for material microstructures. Computational Materials Science, 2008. 43(4): p. 989-999.

17. Michlik, P. and C. Berndt, Image-based extended finite element modeling of thermal barrier coatings. Surface and Coatings Technology, 2006. 201(6): p. 2369-2380. 
18. Legrain, G., et al., An X-FEM and level set computational approach for image-based modelling: Application to homogenization. International Journal for Numerical Methods in Engineering, 2011. 86(7): p. 915-934.

19. Düster, A., et al., The finite cell method for three-dimensional problems of solid mechanics. Computer Methods in Applied Mechanics and Engineering, 2008. 197(45-48): p. 3768-3782.

20. Schillinger, D. and M. Ruess, The Finite Cell Method: A Review in the Context of Higher-Order Structural Analysis of CAD and Image-Based Geometric Models. Archives of Computational Methods in Engineering, 2015. 22(3): p. 391-455.

21. Verhoosel, C.V., et al., Image-based goal-oriented adaptive isogeometric analysis with application to the micro-mechanical modeling of trabecular bone. Computer Methods in Applied Mechanics and Engineering, 2015. 284: p. 138-164.

22. Hollister, S.J. and N. Kikuchi, Homogenization theory and digital imaging: A basis for studying the mechanics and design principles of bone tissue. Biotechnology and Bioengineering, 1994. 43(7): p. 586-596.

23. Garboczi, E.J. and A.R. Day, An algorithm for computing the effective linear elastic properties of heterogeneous materials: Three-dimensional results for composites with equal phase poisson ratios. Journal of the Mechanics and Physics of Solids, 1995. 43(9): p. 1349-1362.

24. Niebur, G.L., et al., Convergence Behavior of High-Resolution Finite Element Models of Trabecular Bone. Journal of Biomechanical Engineering, 1999. 121(6): p. 629-635.

25. Kim, H.J. and C.C. Swan, Voxel-based meshing and unit-cell analysis of textile composites. International Journal for Numerical Methods in Engineering, 2003. 56(7): p. 977-1006.

26. Mishnaevsky Jr, L.L., Automatic voxel-based generation of 3D microstructural FE models and its application to the damage analysis of composites. Materials Science and Engineering: A, 2005. 407(1-2): p. 11-23.

27. Day, A.R., et al., The elastic moduli of a sheet containing circular holes. Journal of the Mechanics and Physics of Solids, 1992. 40(5): p. 1031-1051.

28. Snyder, K.A., E.J. Garboczi, and A.R. Day, The elastic moduli of simple two-dimensional isotropic composites: Computer simulation and effective medium theory. Journal of Applied Physics, 1992. 72(12): p. 5948-5955.

29. Pazdniakou, A. and P.M. Adler, Lattice Spring Models. Transport in Porous Media, 2012. 93(2): p. 243-262.

30. Chen, H. and Y. Liu, The effective elastic and fracture properties of particulate reinforced composites using a new non-local particle method, in 56th AIAA/ASCE/AHS/ASC Structures, Structural Dynamics, and Materials Conference. 2015, American Institute of Aeronautics and Astronautics.

31. Chen, H., Y. Jiao, and Y. Liu, A Nonlocal Lattice Particle Model for Fracture Simulation of Anisotropic Materials. Composites Part B: Engineering, 2016.

32. Chen, H. and Y. Liu, A non-local 3D lattice particle framework for elastic solids. International Journal of Solids and Structures, 2016.

33. Chen, H., E. Lin, and Y. Liu, A novel Volume-Compensated Particle method for 2D elasticity and plasticity analysis. International Journal of Solids and Structures, 2014. 51(9): p. 1819-1833.

34. Hrennikoff, A., Solution of problems of elasticity by the framework method. Journal of Applied Mechanics, 1941. 8: p. A619-A715.

35. Ostoja-Starzewski, M., Lattice Models in Micromechanics. Applied Mechanics Reviews, 2002. 55(1): p. 35-60.

36. Beale, P.D. and D.J. Srolovitz, Elastic fracture in random materials. Physical Review B, 1988. 37(10): p. 5500-5507. 
37. Keating, P.N., Effect of the invariance requirements on the elastic moduli of a sheet containing circular holes. Journal of the Mechanics and Physics of Solids, 1966. 40: p. 1031-1051.

38. Chen, H., et al., A Generalized 2D Non-local Lattice Spring Model for Fracture Simulation. Computational Mechanics, 2014. 54(6): p. 1541-1558.

39. Steen, M. and J.L. Vallés, Determination of in-situ fibre, matrix and interface properties in a composite using tensile tests and an extended shear-lag model. Materials Science and Engineering: A, 1998. 250(2): p. 217-221.

40. Yajing, Y., et al., Molecular dynamics study of pyrolytic carbon interphase in C F /C I preform. Modelling and Simulation in Materials Science and Engineering, 2003. 11(6): p. 897.

41. Amnaya, P.A., C.L. Dimitris, and C.H. Daniel, Modeling of graphene-polymer interfacial mechanical behavior using molecular dynamics. Modelling and Simulation in Materials Science and Engineering, 2009. 17(1): p. 015002.

42. Ostoja-Starzewski, M., P.Y. Sheng, and I. Jasiuk, Damage patterns and constitutive response of random matrix-inclusion composites. Engineering Fracture Mechanics, 1997. 58(5-6): p. 581-606.

43. Ostoja-Starzewski, M. and C. Wang, Linear elasticity of planar delaunay networks: Random field characterization of effective moduli. Acta Mechanica, 1989. 80(1-2): p. 61-80.

44. Chen, H., Y. Jiao, and Y. Liu, Investigating the Microstructural Effect on Elastic and Fracture Behavior of Polycrystals Using a Nonlocal Lattice Particle Model. Materials Science and Engineering: A, 2015. 631: p. 173-180.

45. Jasiuk, I., J. Chen, and M.F. Thorpe, Elastic Moduli of Two Dimensional Materials With Polygonal and Elliptical Holes. Applied Mechanics Reviews, 1994. 47(1S): p. S18-S28.

46. Torquato, S. and Y. Jiao, Dense packings of polyhedra: Platonic and Archimedean solids. Phys. Rev. E, 2009. 80(4): p. 041104.

47. Hill, R., A self consistent mechanics of composite materials. Journal of the Mechanics and Physic of the Solids, 1965(13): p. 213-222.

48. Hashin, Z. and S. Shtrikman, A variational approach to the theory of the elastic behaviour of multiphase materials. Journal of the Mechanics and Physics of Solids, 1963. 11(2): p. 127-140.

49. Torquato, S., Random Heterogeneous Materials: Microstructure and Macroscopic Properties. 2002, New York: Springer-Verlag. 